\title{
Finding the needle in the haystack: BMP9 and 10 emerge from the genome in pulmonary arterial hypertension
}

\author{
Nicholas W. Morrell \\ Affiliation: Dept of Medicine, University of Cambridge School of Clinical Medicine, Cambridge, UK.
}

Correspondence: Nicholas W. Morrell, Division of Respiratory Medicine, Dept of Medicine, University of Cambridge School of Clinical Medicine, Box 157, Addenbrooke's Hospital, Hills Road, Cambridge, CB2 OQQ, UK. E-mail: nwm23acam.ac.uk

@ERSpublications

Two new studies confirm the role of BMP9 mutations as a cause of heritable PAH and provide the first evidence that mutations in BMP10 might also be causal; these results have relevance for the development of new treatments for PAH http://ow.ly/UHmG30nCl3x

Cite this article as: Morrell NW. Finding the needle in the haystack: BMP9 and 10 emerge from the genome in pulmonary arterial hypertension. Eur Respir J 2019; 53: 1900078 [https://doi.org/10.1183/ 13993003.00078-2019].

Evidence is emerging for a circulating axis of vascular protection, in which the release of bone morphogenetic proteins (BMPs) 9 and 10 from the liver and right atrium, respectively, provides tonic quiescent signals to the vascular endothelium [1]. These ligands circulate in the plasma at physiologically active concentrations to stimulate BMP signalling in endothelial cells via a receptor complex comprising the activin receptor-like kinase 1 (ALK1; also known as ACVRL1) and the bone morphogenetic protein receptor type 2 (BMPR2) [2]. We now know that the ALK1/BMPR2 receptor complex, expressed almost exclusively on endothelial cells, responds to BMP9 and BMP10 with high affinity (half-maximal effective concentration (EC50) 50-100 pg. $\mathrm{mL}^{-1}$ ), but does not bind other BMPs [3, 4]. It is well established that heterozygous mutations in the gene encoding BMPR2 are the major genetic cause of pulmonary arterial hypertension (PAH) [5]. In addition, heterozygous mutations in ALK1 are known to be causal in rare cases of $\mathrm{PAH}$, although more commonly cause hereditary haemorrhagic telangiectasia [6]. Taken together, the human genetic evidence suggests a critical BMP9-BMP10/ALK1/BMPR2 axis responsible for maintaining endothelial integrity and function (figure 1). This axis appears to be particularly important in the pulmonary circulation, where the mRNA levels of ALK1 and BMPR2 are higher than in any other organ [7], perhaps explaining the isolated pulmonary vascular involvement in PAH patients. Reduced signalling via this pathway occurs in genetic and non-genetic causes of $\mathrm{PAH}[8,9]$, promoting endothelial dysfunction, increased vascular permeability and apoptosis, disordered angiogenesis and, ultimately, the pathological changes of PAH [10].

Recently, new genes causing PAH were identified in a large European cohort, including heterozygous mutations (missense and premature-truncating variants) in the gene encoding BMP9 (GDF2) as an important cause of PAH [11], reinforcing the central role of the BMP9 signalling pathway. A recent report also demonstrated that patients with portopulmonary hypertension have very low plasma levels of BMP9, which predisposes to PAH in patients with cirrhosis [12]. Furthermore, we have previously demonstrated the therapeutic potential of this pathway, since administration of recombinant BMP9 reversed disease in several preclinical models of PAH [10]. 

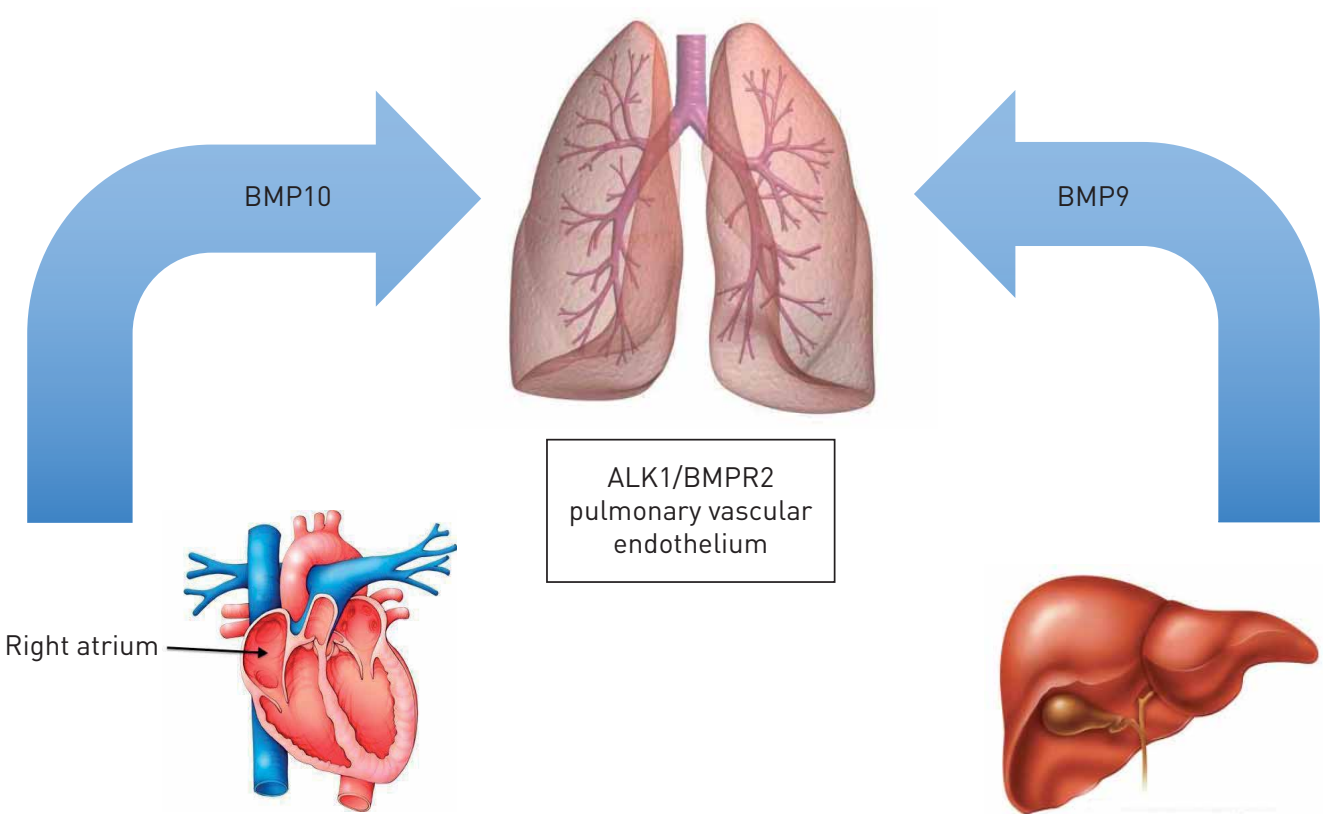

FIGURE 1 Bone morphogenetic protein (BMP)9 and BMP10 circulate in plasma at physiologically active levels and bind selectively to the endothelial receptor complex activin receptor-like kinase 1 (ALK1)/bone morphogenetic protein receptor type 2 (BMPR2), to promote pulmonary vascular health. Disruption of this axis by mutations, loss of circulating levels of ligands, or downregulation of receptors, promotes the development of pulmonary arterial hypertension.

Crucially, Bmp9 knockout mice do not demonstrate a dramatic vascular phenotype (beyond lymphatics), suggesting Bmp9 loss is largely compensated during development. Although circulating Bmp10 levels are unchanged in Bmp9 knockout mice, Bmp10 may circulate as an inactive protein [13]. A recent study reported that BMP9 and BMP10 circulate as a biologically active heterodimer [14], which at first sight conflicts with their distinctive tissue expression patterns. Studies of the cellular origin of liver BMP9 implicate hepatic stellate cells as the major site of production [15], which also express low levels of BMP10 [14], so heterodimers may indeed contribute to circulating activity.

In addition to vascular protection, emerging evidence suggests that BMP9 and BMP10 protect the adult myocardium. BMP10 is highly expressed in the embryonic heart, with expression restricted to the right atrium in adults [16]. Bmp10 knockout mice die by embryonic day 10, due to failed cardiac development [17]. Conversely, Bmp10 overexpression during development increases ventricular hypertrophy and trabeculation [17]. Although Bmp9 knockout mice show normal cardiac development, a recent study reported that BMP9 exerts beneficial effects on cardiac fibrosis and cardiac function in heart failure [18]. These data suggest that loss of BMP9 and BMP10 signalling in the adult heart may predispose to myocardial dysfunction and fibrosis, although studies in the right ventricle in the context of PAH are needed.

In this issue of the European Respiratory Journal, two genetic studies, from France [19] and China [20], provide further insights into the role of BMP9 and BMP10 in the pathobiology of PAH. Both studies independently confirm the occurrence of likely pathogenic mutations in BMP9 in cohorts of patients with idiopathic PAH. Neither study reported a family history of disease in BMP9 mutation carriers, indicating that mutations either occurred de novo or, similar to BMPR2 mutations, disease gene penetrance is reduced. The French study by EYries et al. [19], which included patients with pulmonary veno-occlusive disease (PVOD), used a custom diagnostic gene panel for previously reported PAH/PVOD genes, but also included BMP10, given that it is a close paralogue of BMP9 (65\% amino acid sequence identity). EYRIES et al. [19] confirmed that mutations in BMPR2 are the most common genetic cause of PAH, followed by TBX4, ALK1 (also known as ACVRL1), BMP9 and SMAD9. The genetics and genomics of PAH have been reviewed recently [21]. Importantly, the mutations in $T B X 4$ and $B M P 9$ were highly deleterious truncating mutations, which can be more confidently assigned a pathogenic status than missense mutations involving a single amino acid substitution. EYRIEs et al. [19] further confirmed that biallelic mutations in EIF2AK4 [22] are the most common genetic cause of sporadic PVOD (13.3\% in this series). One patient thought to have PAH by referring clinicians was found to have biallelic EIF2AK4 mutations, allowing them to be 
reclassified as PVOD, confirming the utility of this approach in a previous study [23]. The inclusion of BMP10 in the gene panel allowed the identification of BMP10 mutations in two young patients (aged 11 and 28 years) with severe PAH. One of these mutations is predicted to truncate the protein prior to the growth factor domain, whereas the other was a missense mutation predicted to disrupt the structure of the growth factor domain. Although these mutations look convincingly pathogenic, it will be necessary to determine the functional effects more fully, for example by measurement of mutant BMP10 levels and activity. The 11-year-old patient with the truncating BMP10 mutation also had features of congenital heart disease, in keeping with the known importance of BMP10 during cardiac development. A further point of interest from this French cohort is that, of $11 \mathrm{PAH}$ patients reporting a family history of disease, only six received a genetic diagnosis from the gene panel testing (and all of these six were BMPR2 mutations). Although the panel did not include all the most recently reported PAH genes, this finding implies that a significant number of patients have a genetic diagnosis that is yet to be identified. Such patients could be offered further unbiased genetic testing by whole exome or whole genome sequencing to look for novel causal PAH genes.

The study from China by WANG et al. [20] undertook whole exome or whole genome sequencing of DNA from 331 adult- and childhood-onset idiopathic PAH cases compared with 10508 controls. The proportion of PAH patients found to have a genetic diagnosis based on the established PAH genes [21] was higher in this cohort (36.3\%) than generally reported. Similar to the French study by EYRIES et al. [19], four cases of biallelic EIF2AK4 mutations were picked up in PAH patients, allowing reclassification of these patients as PVOD. In PAH patients, mutations in BMPR2 (19.5\%) were the most common, followed by ALK1 (6\%) and TBX4 (4\%), with other genes each accounting for $<1 \%$ of the entire cohort. Having determined the frequency of mutations in these established genes, WANG et al. [20] undertook a casecontrol analysis with discovery and replication cohorts, to look for overrepresentation of rare variants in other genes that were significant at the genome-wide level. This resulted in the identification of predicted deleterious heterozygous mutations in BMP9 in 6.7\% of cases (21 distinct mutations in 22 cases), making mutations in BMP9 the second most common cause of PAH in this Chinese cohort. Mutations included three nonsense mutations, two frameshifts and one that disrupted the translational start site. The remaining 15 mutations were missense. The majority (14 out of 15) of missense mutations were entirely absent from 6512 control individuals of Chinese ancestry and were predicted to be deleterious by commonly used algorithms. To overcome the potential pitfall of calling rare missense variants pathogenic without functional experimental data, WANG et al. [20] showed that a subset of missense mutations leads to reduced secretion of the mature BMP9 protein from transfected cells, similar to a previous report [11], or demonstrated reduced signalling activity in a reporter cell line. Finally, WANG et al. [20] measured plasma BMP9 by ELISA in 19 BMP9 mutation carriers compared with age- and sex-matched idiopathic PAH patients and healthy controls. Median plasma BMP9 levels were significantly lower in idiopathic PAH patients and were even lower in patients carrying BMP9 mutations, albeit with a wide range of levels and overlapping values between groups. These measurements provide evidence that the missense mutations in BMP9 actually affect plasma levels of the ligand, further increasing the burden of proof that they are pathogenic. Measurements of plasma BMP9 activity would provide further confirmation.

Taken together, these reports provide important confirmation of the role of BMP9 mutations as a cause of heritable PAH and provide the first evidence that mutations in BMP10 might also be causal. Further confirmation of the BMP10 finding is required, accompanied by functional studies. Do these findings have relevance for the development of new treatments for PAH? Certainly, the finding of patients with mutations in BMP9, with reduced BMP9 levels, indicates that supplementing BMP9 in these individuals would be an attractive precision medicine approach. Indeed, exogenous BMP9 could provide a direct, targeted approach to restore physiological ALK1/BMPR2 endothelial signalling in genetic and non-genetic forms of PAH [10], if it could be administered safely. Such approaches are now under active development.

Conflict of interest: N.W. Morrell reports personal fees from Morphogen-IX, GSK and Actelion, outside the submitted work; in addition, he holds patents on the use of BMP9 and BMP10 as therapies, both pending and licensed to Morphogen-IX.

Support statement: N.W. Morrell is funded by a British Heart Foundation Personal Chair Award. Funding information for this article has been deposited with the Crossref Funder Registry.

\section{References}

1 David L, Mallet C, Keramidas M, et al. Bone morphogenetic protein-9 is a circulating vascular quiescence factor. Circ Res 2008; 102: 914-922.

2 Li W, Salmon RM, Jiang H, et al. Regulation of the ALK1 ligands, BMP9 and BMP10. Biochem Soc Trans 2016; 44: 1135-1141 
3 Upton PD, Davies RJ, Trembath RC, et al. Bone morphogenetic protein (BMP) and activin type II receptors balance BMP9 signals mediated by activin receptor-like kinase-1 in human pulmonary artery endothelial cells. J Biol Chem 2009; 284: 15794-15804.

4 David L, Mallet C, Mazerbourg S, et al. Identification of BMP9 and BMP10 as functional activators of the orphan activin receptor-like kinase 1 (ALK1) in endothelial cells. Blood 2007; 109: 1953-1961.

5 International PPH Consortium, Lane KB, Machado RD, et al. Heterozygous germline mutations in BMPR2, encoding a TGF- $\beta$ receptor, cause familial primary pulmonary hypertension. Nat Genet 2000; 26: 81-84.

6 Trembath RC. Mutations in the TGF-beta type 1 receptor, ALK1, in combined primary pulmonary hypertension and hereditary haemorrhagic telangiectasia, implies pathway specificity. J Heart Lung Transplant 2001; 20: 175.

7 The Broad Institute of MIT and Harvard. The Genotype-Tissue Expression (GTEx) Portal. https://gtexportal.org/ Date last accessed: February 1, 2019.

8 Morrell NW, Yang X, Upton PD, et al. Altered growth responses of pulmonary artery smooth muscle cells from patients with primary pulmonary hypertension to transforming growth factor- $\beta_{1}$ and bone morphogenetic proteins. Circulation 2001; 104: 790-795.

9 Long L, Crosby A, Yang X, et al. Altered bone morphogenetic protein and transforming growth factor- $\beta$ signaling in rat models of pulmonary hypertension: potential for activin receptor-like kinase- 5 inhibition in prevention and progression of disease. Circulation 2009; 119: 566-576.

10 Long L, Ormiston ML, Yang X, et al. Selective enhancement of endothelial BMPR-II with BMP9 reverses pulmonary arterial hypertension. Nat Med 2015; 21: 777-785.

11 Gräf S, Haimel M, Bleda M, et al. Identification of rare sequence variation underlying heritable pulmonary arterial hypertension. Nat Commun 2018; 9: 1416.

12 Nikolic I, Yung LM, Yang P, et al. Bone morphogenetic protein 9 is a mechanistic biomarker of portopulmonary hypertension. Am J Respir Crit Care Med 2018; in press [https://doi.org/10.1164/rccm.201807-1236OC].

13 Ricard N, Ciais D, Levet S, et al. BMP9 and BMP10 are critical for postnatal retinal vascular remodeling. Blood 2012; 119: 6162-6171.

14 Tillet E, Ouarné M, Desroches-Castan A, et al. A heterodimer formed by bone morphogenetic protein 9 (BMP9) and BMP10 provides most BMP biological activity in plasma. J Biol Chem 2018; 293: 10963-10974.

15 Breitkopf-Heinlein K, Meyer C, König C, et al. BMP-9 interferes with liver regeneration and promotes liver fibrosis. Gut 2017; 66: 939-954.

16 Chen H, Shi S, Acosta L, et al. BMP10 is essential for maintaining cardiac growth during murine cardiogenesis. Development 2004; 131: 2219-2231.

17 Zhang $\mathrm{W}$, Chen $\mathrm{H}$, Wang $\mathrm{Y}$, et al. Tbx20 transcription factor is a downstream mediator for bone morphogenetic protein-10 in regulating cardiac ventricular wall development and function. J Biol Chem 2011; 286: 36820-36829.

18 Morine KJ, Qiao X, York S, et al. Bone morphogenetic protein 9 reduces cardiac fibrosis and improves cardiac function in heart failure. Circulation 2018; 138: 513-526.

19 Eyries M, Montani D, Nadaud S, et al. Widening the landscape of heritable pulmonary hypertension mutations in paediatric and adult cases. Eur Respir J 2019; 53: 1801371.

20 Wang X-J, Lian T-Y, Jiang X, et al. Germline BMP9 mutation causes idiopathic pulmonary arterial hypertension. Eur Respir J 2019; 53: 1801609.

21 Morrell NW, Aldred MA, Chung WK, et al. Genetics and genomics of pulmonary arterial hypertension. Eur Respir J 2019; 53: 1801899.

22 Eyries M, Montani D, Girerd B, et al. EIF2AK4 mutations cause pulmonary veno-occlusive disease, a recessive form of pulmonary hypertension. Nat Genet 2014; 46: 65-69.

23 Hadinnapola C, Bleda M, Haimel M, et al. Phenotypic characterization of EIF2AK4 mutation carriers in a large cohort of patients diagnosed clinically with pulmonary arterial hypertension. Circulation 2017; 136: 2022-2033. 